\title{
Exosome-mediated communication in the tumor microenvironment contributes to hepatocellular carcinoma development and progression
}

\author{
Qin Wu $\mathrm{W}^{1,2}$, Lingyun Zhou ${ }^{1,2}$, Duoduo Lv ${ }^{1,2}$, Xia Zhu ${ }^{1,2}$ and Hong Tang ${ }^{1,2^{*}}$
}

\begin{abstract}
The tumor microenvironment (TME) is an essential intrinsic portion of hepatocellular carcinoma (HCC) for the regulation of its origination, development, invasion, and metastasis. As emerging components of the tumor-host interaction, exosomes are increasingly recognized as professional carriers of information in TME and as pivotal molecular entities involved in tumorigenic microenvironment setup. However, much remains unknown about the role of the exosome communication system within TME in the development and progression of HCC. In this review, we focus on the roles and probable mechanisms of TME in HCC and show the exosome-based immune regulation in TME to promote HCC. Multiple processes are involved in HCC, including tumor survival, growth, angiogenesis, invasion, and metastasis. We also discuss the specific roles of exosomes in HCC processes by molding hospitable TME for HCC, such as providing energy, transmitting protumor signals, and evading inhibitory signals. In addition, exosomes induce angiogenesis by changing the biological characteristics of endothelial cells and directly regulating proangiogenic and propermeability factors. Furthermore, exosomes may lead to HCC metastatic invasion by epithelial-mesenchymal transformation, extracellular matrix degradation, and vascular leakage. Finally, we summarize the therapeutic usage of exosomes in the HCC microenvironment and attempt to provide a theoretical reference for modern antitumor agents designed to target these mechanisms.
\end{abstract}

Keywords: Exosomes, Tumor microenvironment, Hepatocellular carcinoma, Immune regulation, Therapy

\section{Background}

The tumor microenvironment (TME) is the cellular environment in which the tumor develops. Apart from the tumor cells, the TME includes various cell types, extracellular matrix (ECM), growth factors, proteolytic enzymes and their inhibitors, and signaling molecules $[1,2]$. TME influences tumor growth, metastasis, and ultimately prognosis. Therefore, the fundamental role of TME is to dynamically interact with malignant cells [3]. The TME contributes significantly to the pathogenesis of hepatocellular carcinoma (HCC). Indeed, by offering, inhibiting, or

\footnotetext{
* Correspondence: htang6198@hotmail.com

${ }^{1}$ Center of Infectious Diseases, West China Hospital, Sichuan University, Chengdu, China

${ }^{2}$ Division of Infectious Diseases, State Key Laboratory of Biotherapy and Center of Infectious Diseases, West China Hospital, Sichuan University, Chengdu, China
}

stimulating growth signals, this TME is an essential modulator of $\mathrm{HCC}$ development and progression and a source for identifying targets for potential therapeutic agents [4].

The interactions of HCC cells with the surrounding TME are based on complex systemic networks. In addition to direct cell-to-cell contact, intercellular communication through secreted factors plays a key role in intercellular signaling. Among these secreted factors, exosomes are the major components of extracellular vesicles (EVs), which range in size from 30 to $150 \mathrm{~nm}$. EVs originate from multivesicular bodies (MVBs) and are generated by all cell types $[5,6]$. Upon early to late endosome maturation, biomolecules are endocytosed and transported into early endosomes. In late endosomes, intraluminal vesicles (ILVs) are formed by inward budding of the endosomal membrane and result in a large MVB. MVBs can fuse with the plasma membrane, and the ILVs released into the extracellular 
space are referred to as "exosomes" [7, 8]. However, studies on the genesis and release of exosomes have revealed that apart from the sorting of cargo molecules, the procedure is tightly associated with energy mediators, such as SNAREs, Rabs, and Ras GTPases [9]. Exosomes are generated in the form of endocytosis, exocytosis, protein transport, and protein sorting. During this process, exosomes are packed with lipids, proteins, DNA, mRNA, miRNA, and other ncRNAs $[5,10]$, which are horizontally transferred from donor to recipient cells. Exosomes can carry biomolecules from tissues to body fluids [11-15]. These properties contribute to the role of exosomes in intercellular communication, i.e., shuttling of signaling molecules between nearby and remote cells [16-18]. The surface of the exosome contains a large number of molecules related to antigen presentation. In vivo and in vitro, exosomes have similar effects as antigen-presenting cells, which can induce and enhance immune responses. Exosomes have widely dissimilar sizes and contents and are heterogeneous in biological effects and targeting specificities. Thus, exosomes have attracted attention as important vehicles for specific signals in tumor progression, metastasis, immune modulation, angiogenesis, and tissue regeneration [19].

In the liver, exosomes are secreted by three main cell types: liver epithelia (i.e., hepatocytes and cholangiocytes), immune cells (i.e., T and B cells, dendritic cells, and NK cells), and nonparenchymal liver cells (e.g., liver stellate cells) [20-22]. Further evidence suggests a role for exosomes derived from different liver cells in the intracellular communication for the coordination of cell behaviors proper functioning. For example, exosomes derived from hepatocytes and cholangiocytes are important mediators of proliferation processes [20, 23]. T celland B cell-derived exosomes are involved in inflammation [24]. Exosomes derived from hepatic stellate cells (HSCs) may be involved in the pathogenesis of liver fibrosis [25]. Furthermore, primary hepatocyte-derived exosomes promote the activation of stellate cells, which in turn participate in liver disease progression [26]. Moreover, lipid-induced EVs derived from hepatocytes also cause an inflammatory macrophage phenotype [27]. Exosomes derived from the cells of other organs and tissues are involved in various types of liver disease [21]. For example, exosomes are involved in the progression of viral infections, including viral transmission, immune response, and antiviral effect [28, 29]; several studies have suggested that EVs increase with alcoholic hepatitis and show upregulation, even with excessive alcohol consumption $[30,31]$. The role of exosomes in liver fibrosis by regulating connective tissue growth factor 2-dependent fibrogenesis in HSCs has also been reported [32].

However, what is the role of exosomes in HCC, and how is TME remolded by exosomes? With these considerations, we will focus here on the role of exosomes in setting up and modifying the $\mathrm{HCC}$ microenvironment that encourages tumor development and progression. We will discuss the specific mechanisms by which exosome-based communication regulates the immune response and benefits tumor survival, growth, angiogenesis, and metastasis (Fig. 1). We will also summarize the potential clinical applications of exosomes as therapeutic modalities.

\section{Role of the tumor microenvironment in promoting the development and progression of HCC}

Despite advances in prevention, screening, and novel diagnostic and therapeutic technologies, HCC therapy has encountered a bottleneck. The 5-year survival rate of patients with $\mathrm{HCC}$ is still $<20 \%[33,34]$, which indicates that HCC remains a highly lethal disease. An emerging concept is that the TME plays an important role in initiating and maintaining carcinogenesis [35]. The TME is a varying environment that describes the behavior of cancer. The TME is the cellular environment that tumor cells need for survival, growth, proliferation, and metastasis [36]. It is a complex and heterogeneous system that is orchestrated by two major groups of cellular and noncellular elements.

The cellular elements in the TME, such as HSCs, fibroblasts (cancer-associated fibroblasts or CAFs), immune cells (T lymphocytes, B lymphocytes, NK cells, natural killer T cells, and tumor-associated macrophages or TAMs), and endothelial cells (ECs), play a critical role in tumor-stromal interactions that can modulate biological activities of HCC. A study has shown that activated HSCs infiltrate the stroma of HCC and are associated with tumor proliferation [37]. One of the mechanisms is that HSCs reduce HCC central necrosis [38]. CAFs are the best studied stromal cells and are defined as loose fibroblasts found within the tumor mass. CAFs maintain the stem cell-like properties of HCC cells by secreting IL-6 [39] and promote tumor invasion and metastasis by secreting matrix metalloproteinase- 9 (MMP-9) [40]. Immune cells are commonly described to restrain tumor development; however, when they intimately interact with transformed cells in the TME, these cells can alternatively propagate tumor progression [41, 42]. For example, the deficiencies and malfunction of immune cells can introduce an immunosuppressive microenvironment for tumor cells to achieve immune tolerance and evasion. ECs in TME are important suppliers of nutrients, oxygen, and various growth factors for HCC development.

The noncellular elements include growth factors, such as transforming growth factor- $\beta$ (TGF- $\beta$ ), insulin-like growth factor (IGF), fibroblast growth factor (FGF), hepatocyte growth factor (HGF), and vascular endothelial growth factor (VEGF). The remaining noncellular elements also include proteolytic enzymes, ECM, and inflammatory cytokines. These factors can provide a pliable environment that encourages further HCC growth and propagation. TGF- $\beta$ 


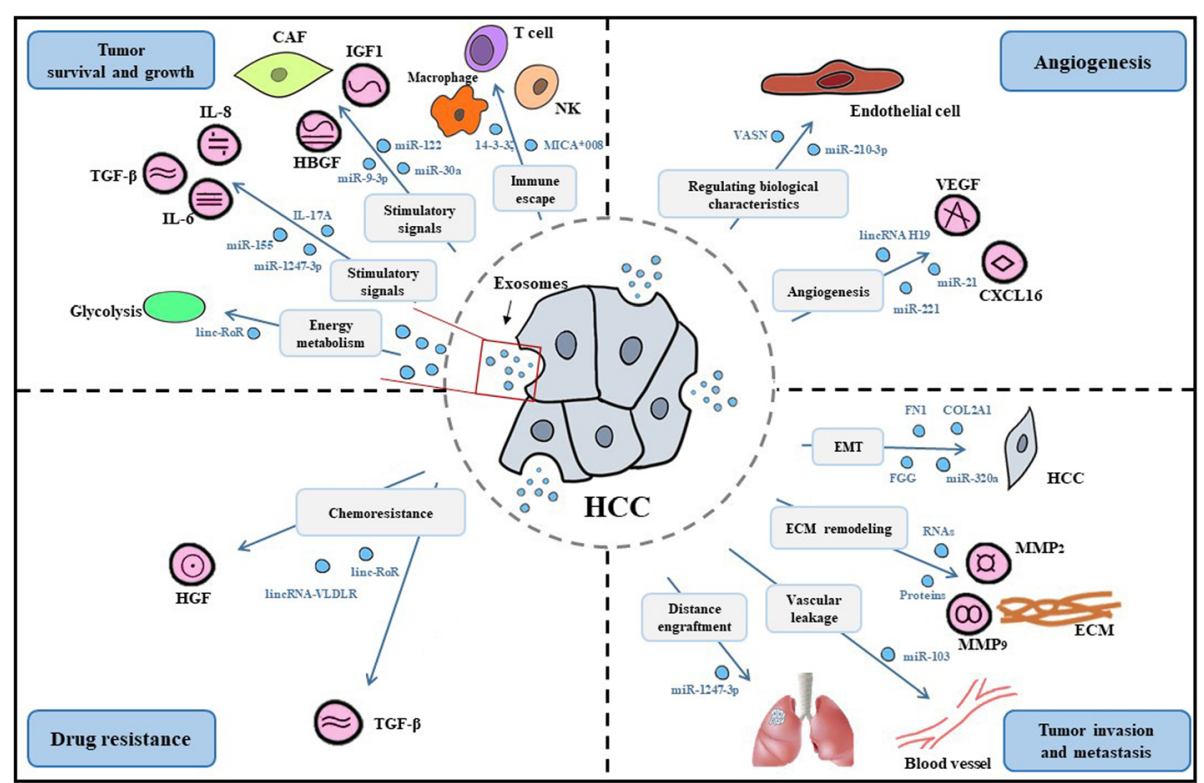

Fig. 1 The functions of exosomes within TME in facilitating the development and progression of HCC. Exosome-mediated immune regulation is involved in modulation of the TME with immunosuppressive and tolerogenic characteristics. Multiple processes are involved in HCC, including tumor survival, growth, angiogenesis, invasion, and metastasis. The specific mechanisms involved in molding the TME for HCC include energy metabolism regulation, stimulatory signal supply, and inhibitory signal circumvention. In addition, exosomes induce angiogenesis by changing the biological characteristics of endothelial cells and directly regulating proangiogenic and propermeability factors. Additionally, exosomes may lead to HCC metastatic invasion by EMT, ECM degradation, and vascular leakage. Finally, the association between exosomes and drug resistance suggests the involvement of complex mechanisms of chemoresistance in the TME

is an essential component of liver disease by participating in responses to surroundings and acting as a continual target for tumor-derived signals [43]. IGFs are mainly synthesized in the liver and are functionally related to HCC proliferation [44, 45]. Another important growth factor, FGF, is characterized by multiple functions that can mediate cell proliferation, angiogenesis, wound healing, and tissue repair [46]. HGF in the TME can promote malignant cell growth and survival [47]. Abnormal ECM in the TME can play a critical role in promoting angiogenesis and inflammation [48]. Many inflammatory cytokines, such as IL-6 and IL-8, as well as MMP2 and MMP-9, participate in regulating the inflammatory microenvironment and contribute to the increased migratory and invasive potential of hepatocytes, thus facilitating cancer metastasis $[49,50]$.

Together, these data suggest that TME has a determinate protumorigenic effect and works as a booster in HCC progression and metastasis. TME has been considered to modulate the survival and growth of cell lines by providing inhibitory or stimulatory signals [4]. However, how are signals transmitted to and received from the TME? In recent years, growing evidence indicates that exosomes are important vehicles of specific signals in physiological scenarios [5]. In addition, exosomes have been increasingly studied as novel intercellular communication mediators for tumor onset and progression in TME [19].

\section{Exosome-mediated immune regulation in the HCC microenvironment}

The immune microenvironment is shaped by intricate interactions between tumor cells and the host immune response. The evolution of cancer is strongly related to the tumor immune microenvironment. HCC shows a high degree of malignancy, and its poor overall survival outcome is due to the collapse of immune surveillance, which is closely associated with the suppression of host immune responses. Mounting evidence indicates that exosome-derived interactions are involved in the modulation of the microenvironment with immunosuppressive and tolerogenic characteristics. By regulating immune responses, tumors can evade immune destruction, and progression is ensured in this protumorigenic immune environment [51-54]. Pioneering work by Wang et al. [55] demonstrated that $\mathrm{T}$ cells could swallow HCCderived exosomes containing 14-3-3 14-3-3 $\zeta$ was upregulated in HCC cells and in tumorinfiltrating $\mathrm{T}$ lymphocytes (TILs). This process was related to impaired activation (CD69 expression), proliferation (Ki67 expression), and antitumor functions. In addition, $14-3-3 \zeta$ overexpression inhibits the vitality and proliferation of peripheral blood CD3 $+\mathrm{T}$ cells, promoting the differentiation of naive $\mathrm{T}$ cells from effector $\mathrm{T}$ cells to regulatory $\mathrm{T}$ cells. These findings suggested that HCC-derived exosomes containing 14-3-3 $\zeta$ could inhibit 
the antitumor functions of TILs in the HCC microenvironment. NKG2D is a C-type lectin-like activating receptor expressed on all NK cells. Evidence has shown that NKG2D binding to its ligands mediates immune system activation and plays an important role in cancer immune surveillance [56, 57]. In fact, exosomes secreted by liver tumor cells induce NKG2D downregulation on NK cell surfaces, which leads to impaired cytotoxic function and is beneficial for HCC immune escape and progression [58]. Rao et al. demonstrated that HCC-derived exosomes can trigger DC-mediated immune responses by serving as vectors for a variety of antigens and remold the HCC tumor immune microenvironment [59]. Within the TME, tumor-derived exosomes play a key role in the polarization status of macrophages. In this setting, macrophages are observed to shift to a protumorigenic macrophage phenotype (M2 polarization), leading to tumor angiogenesis and metastasis [60,61]. Melatonin is a hormone with certain cytotoxic and immunomodulatory effects, and this hormone can inhibit the functions of tumors. A recent study found that after treatment of hepatocellular carcinoma cells with melatonin, HCC-derived exosomes can alter the immunosuppressive status through the STAT3 pathway in macrophages [62].

HCC-derived exosomes can modulate the phenotype and function of immunocytes that can be beneficial for tumor cells to escape immune destruction. In turn, exosomes secreted by immunocytes in TME can affect antitumor immune responses. Mast cells can be stimulated by hepatitis $\mathrm{C}$ virus E2 envelope glycoprotein (HCV-E2) to secrete a large number of exosomes rich in miR-490. These exosomes are transferred into HCC cells and inhibit the metastasis of tumor cells via inhibiting the ERK1/2 pathway [63]. Exosomes derived from TAMs indisputably play a key role in immune suppression, angiogenesis, and tumor progression. For example, human macrophages can transfer exosomes with miR-142 and miR-223 to liver cells and inhibit the proliferation or growth of tumor cells [64]. In conclusion, HCC is characterized by the ability to employ different strategies to evade host immune surveillance, and exosomes are important promoters to alternatively propagate tumor progression by immune regulation.

\section{Exosomes mold the tumor microenvironment for HCC development and progression}

Tumor survival and growth: exosome-based communication in the HCC microenvironment functions as a determinant factor

Cancer development and progression relies on its complicated and heterogeneous microenvironment, which consists of a network of cellular and acellular constituents. This process is impacted by exosome-mediated transfer within the TME. The role and mechanisms of exosomes in the HCC microenvironment participating in multiple steps during tumor development and progression will be highlighted in this review (Table 1).

\section{Regulating energy metabolism}

Tumor cells produce hypoxia-inducible factor (HIF) as an important mediator of tumor metabolism, which regulates the expression of target genes, such as erythropoietin, iron metabolism-related genes, VEGF, GLUT1, glycolytic enzymes, heme oxygenase $\mathrm{HO} 2-1$, and inducible nitric oxide synthase. Thus, tumor cells can reprogram energy metabolism under hypoxic conditions and achieve malignant proliferation [90]. Takahashi et al. [65] found that under hypoxic conditions, hepatocellular carcinoma cells promote the secretion of linc-ROR-containing exosomes and escalate the expression of miRNA-145, HIF- $1 \alpha$, and its downstream regulatory protein pyruvate dehydrogenase kinase isozyme 1 (PDK1). Furthermore, knockdown of linc-RoR has a noteworthy effect on the recovery of the expression of these three proteins. PDK1 is a hypoxia stress protein that regulates mitochondrial function and enhances glycolysis by reducing the entry of pyruvate into the tricarboxylic acid cycle. Therefore, HCC-derived exosomes can activate the microRNA-145/HIF-1 $\alpha /$ PDK1 pathway, which may enhance the glycolysis process and resist a hypoxic environment. However, further investigation is required to determine the specific mechanisms.

\section{Providing stimulatory signals}

Exosomes secreted by HCC regulate the surrounding microenvironment and create conditions conducive to tumor growth and development. The multifarious characteristics of growth factors in HCC have been well studied by many researchers. The development of HCC is associated with TGF- $\beta$ overexpression [91]. Furthermore, TGF- $\beta$ overexpression in HCC patients gives significant advantages to tumor growth by facilitating the advancement of a favorable microenvironment [92]. Takayuki et al. demonstrated that exosomes packed with miRNA secreted by HCC cells promote tumor motility, invasion, and dissemination by inducing the expression of TGF- $\beta$ and TGF- $\beta$ activated kinase-1 [12]. An inflammatory environment effectively facilitates HCC formation and development. In this setting, exosomes have been shown to play an important role in mediating the regulation of the inflammatory microenvironment to promote cancer progression and metastasis. For instance, when exposed to arsenite, HCC cells produce miR-155-rich exosomes that enhance inflammation and positively correlate with IL-6 or IL-8 levels [67]. Another example involves the bidirectional communication between HCC and its exosome-mediated inflammatory microenvironment, that is, while the inflammatory microenvironment promotes tumorigenesis, the tumor also creates an inflammatory environment that promotes its 
Table 1 The roles of exosomes within TME in HCC development and progression

\begin{tabular}{|c|c|c|c|c|}
\hline Exosomal cargos & Regulation & Biological function & Mechanism & Reference \\
\hline $14-3-3 \zeta$ & Increased & Impair antitumor function & $\begin{array}{l}\text { Inhibit the antitumor functions of TILs and } \\
\text { the vitality and proliferation of peripheral } \\
\text { blood CD3+ T cells }\end{array}$ & {$[55]$} \\
\hline $\mathrm{MICA}^{*} 008$ & Increased & Impair cytotoxic function & $\begin{array}{l}\text { Induce NKG2D downregulation on NK } \\
\text { cell surface }\end{array}$ & {$[58]$} \\
\hline HCC antigens & Increased & Promote immune responses & Activate immune response mediated by DCs & {$[59]$} \\
\hline Not mentioned & Unvaried & Regulate immunosuppression & $\begin{array}{l}\text { Alter the immunosuppressive status through } \\
\text { STAT3 pathway in macrophages }\end{array}$ & [62] \\
\hline miR-490 & Increased & Inhibit metastasis & $\begin{array}{l}\text { Mast cells are stimulated by HCV-E2 and secrete } \\
\text { exosomes to inhibit the ERK1/2 pathway }\end{array}$ & [63] \\
\hline miR-142, miR-223 & Increased & Inhibit proliferation & $\begin{array}{l}\text { Decrease reporter protein expression and } \\
\text { endogenously express stathmin-1 and insulin-like } \\
\text { growth factor-1 receptor }\end{array}$ & {$[64]$} \\
\hline \multirow[t]{2}{*}{ linc-RoR } & \multirow[t]{2}{*}{ Increased } & Regulate energy metabolism & $\begin{array}{l}\text { Activate microRNA-145/HIF-1a/PDK1 pathway } \\
\text { and enhance the glycolysis process }\end{array}$ & {$[65]$} \\
\hline & & Induce chemoresistance & $\begin{array}{l}\text { Activate TGF- } \beta \text { signaling and promote colony } \\
\text { formation of CD 133+ T-IC }\end{array}$ & {$[66]$} \\
\hline miRNA & Increased & Promote migration and invasion & Induce TGF- $\beta$ and TAK1 expression & {$[12]$} \\
\hline miR-155 & Increased & Promote formation and development & $\begin{array}{l}\text { Promote inflammation and positively correlate } \\
\text { with IL-6 or IL-8 levels }\end{array}$ & {$[67]$} \\
\hline miR-1247-3p & Increased & Promote metastasis & $\begin{array}{l}\text { Target B4GALT3 and activate } \beta 1 \text {-integrin-NF-KB } \\
\text { pathway }\end{array}$ & {$[50]$} \\
\hline miR-30a & Decreased & Promote proliferation and metastasis & Mediate Beclin 1 and Atg5-dependent autophagy & {$[68]$} \\
\hline miR-320a & Decreased & Promote metastasis & $\begin{array}{l}\text { Target PBX3 and MAPK pathway, induce EMT, } \\
\text { and upregulate CDK2 and MMP-2 expression }\end{array}$ & [69] \\
\hline \multirow[t]{2}{*}{ miR-122 } & Decreased & Promote proliferation & $\begin{array}{l}\text { IGF1 prevents intercellular exosomal transfer } \\
\text { of miR-122 }\end{array}$ & {$[70]$} \\
\hline & Increase & Induce chemosensitivity & Induce chemosensitivity (5-FU and sorafenib) & {$[71]$} \\
\hline miR-9-3p & Decreased & Promote proliferation & Regulate HBGF-5 and ERK1/2 expression & {$[72]$} \\
\hline VASN & Increased & Promote HUVECs cells migration & Not mentioned & [73] \\
\hline RNAs, miRNAs & Polytropic & $\begin{array}{l}\text { Associated with the degree of } \\
\text { lumen formation }\end{array}$ & Not mentioned & {$[74]$} \\
\hline miR-210-3p & Increased & Increase angiogenesis & $\begin{array}{l}\text { Inhibit the expression of SMAD4 and STAT6 } \\
\text { in ECS }\end{array}$ & {$[75]$} \\
\hline lincRNA H19 & Increased & Increase angiogenesis & $\begin{array}{l}\text { Increase VEGF release and the production } \\
\text { of VEGF-R1 }\end{array}$ & {$[76]$} \\
\hline $\operatorname{miR}-221$ & Increased & Increase angiogenesis & $\begin{array}{l}\text { Activate SAND/NF-KB pathway and upregulate } \\
\text { CXCL16 expression }\end{array}$ & {$[77,78]$} \\
\hline miR-21 & Increased & Increase angiogenesis & Activate the STAT3NEGF pathway & {$[77,79]$} \\
\hline Not mentioned & Not mentioned & Induce chemoresistance & $\begin{array}{l}\text { Activate HGF/c-Met/Akt pathways and restrain } \\
\text { apoptosis }\end{array}$ & {$[80]$} \\
\hline Fibronectin1 COL2A1, FGG & Increased & Promote metastasis & Induce either partial or total EMT & {$[81]$} \\
\hline RNAs, proteins & Polytropic & Promote migration and invasion & $\begin{array}{l}\text { Activate PI3K/AKT and MAPK pathways in MIHA } \\
\text { and increase MMP-2 and MMP-9 secretion }\end{array}$ & {$[82]$} \\
\hline miR-103 & Increased & Promote metastasis & Inhibit the expression of VE-Cad, p120, and ZO-1 & {$[83]$} \\
\hline AFP & Increased & $\begin{array}{l}\text { Promote immune responses; } \\
\text { Inhibit proliferation }\end{array}$ & $\begin{array}{l}\text { Enhance CD8+ T lymphocytes response, improve } \\
\text { IFN- } \gamma \text { and IL-2 expression }\end{array}$ & {$[84]$} \\
\hline HSP & Increased & Promote immune responses & $\begin{array}{l}\text { Improve tumor immunogenicity and induce } \\
\text { NK cell responses }\end{array}$ & {$[85]$} \\
\hline PD-L1 & Increased & Promote proliferation & Suppress $T$ cell activation in the draining & {$[86]$} \\
\hline
\end{tabular}


Table 1 The roles of exosomes within TME in HCC development and progression (Continued)

\begin{tabular}{|c|c|c|c|c|}
\hline Exosomal cargos & Regulation & Biological function & Mechanism & Reference \\
\hline miR-26a & Increased & Inhibit proliferation & $\begin{array}{l}\text { Bind to HepG2 cells via the scavenger receptor } \\
\text { class B type 1-Apo-A1 complex }\end{array}$ & {$[87]$} \\
\hline Doxorubicin & Increased & Inhibit proliferation & $\begin{array}{l}\text { imDCs were engineered to express Lamp2b fused } \\
\text { with } v \text { integrin-specific iRGD peptide }\end{array}$ & [88] \\
\hline lincRNA-VLDLR & Increased & Induce chemoresistance & Increase ABCG2 expression and restrain apoptosis & [89] \\
\hline
\end{tabular}

own development. High-metastatic $\mathrm{HCC}$ cells secrete exosomes with miR-1247-3p, activating $\beta 1$-integrin-NF-kB signaling in fibroblasts by directly targeting B4GALT3. In fact, activated CAFs further promote pleiotropic actions, such as secreting proinflammatory cytokines (IL-6 and IL-8) that govern the progression of cancer [50].

Conversely, exosomes secreted from surrounding nontumor cells can transmit growth signals, promoting tumor expansion and aggressiveness. When liver injury occurs, hepatic exosomes activate HSCs through increasing IL-17A production [93]. IL-17A is associated with the progression of $\mathrm{HCC}$ [94]. In vitro and in vivo studies have demonstrated that HSC-conditioned media prominently promoted HCC cell proliferation and development: activated HSCs infiltrate the stroma of $\mathrm{HCC}$ and are involved in the proliferation of HCC cells [37]; in line with this finding, co-cultured HSCs and HCC cells can promote tumor growth and invasiveness in nude mice [38]. These findings indicate that signals can be transmitted to HSCs via exosomes, potentially promoting the onset and progression of HCC. However, the specific mechanisms by which exosomes trigger healthy stromal cells to promote the malignant behavior of cancer cells require further investigation.

\section{Eluding inhibitory signals}

In the past decade, there has been increasing emphasis on the potential role of exosome-mediated HCC to evade inhibitory signals, thereby promoting the malignant behavior of cancer cells. miR-30a, as a cancer suppressor, has been demonstrated to inhibit migration and invasion in HCC [95]. Exosomes packed with miR-30a derived from HSCs and LX-2, which were activated by TGF- $\beta 1$, could notably be downregulated [96]. A recent study showed that HCC promotes vascular invasion, metastatic potential, and recurrent disease by downregulating miR-30a in tissues and cell lines [68]. In addition, CAF-derived exosomes overexpressing miR-320a can inhibit tumorigenesis, which implies that a possible cause of HCC progression mediated by CAFs is linked with a deficiency in antitumor miR-320a in CAF-derived exosomes [69]. miR-122 has liver-specific antiproliferative characteristics [97]. Basu et al. detected the intercellular transfer of miR-122 via exosomes, but exosomal transfer was prevented by IGF1 in HCC, suggesting that the expression of exosomal miR-122 is prevented to ensure tumor proliferation [70]. Similarly, $\mathrm{HCC}$ can reduce the expression level of serum exosomes with miR-9$3 \mathrm{p}$ to promote tumor cell viability and proliferation [72]. Most studies on the relevance of miRNA in cancer biology have been performed in vitro, which has some limitations. However, numerous studies suggest that exosome-derived miRNAs can mediate the biological information transmission between $\mathrm{HCC}$ and TME and participate in the biological process of $\mathrm{HCC}$ in multiple forms.

\section{Exosome-induced angiogenesis: the tumor microenvironment has a complex impact}

Active angiogenesis has been suggested as the leading cause of rapid tumor growth, early metastasis, and poor survival [98]. In addition, exosomes regulate intercellular communication through proteins, nucleic acids, and other cargo to alter the TME, inducing the formation of vascular lumens and ultimately promoting the malignant proliferation and invasive phenotype of tumor cells.

\section{Regulating biological characteristics of endothelial cells}

ECs are recognized as essential components of TME by providing a conduit to nutrients, oxygen, and various growth factors in the course of tumor development. Several proteins, such as VEGF and angiopoietin, have been implicated in regulating the biological and physical characteristics of ECs. Additionally, tumor cell-derived exosomes containing functional proteins and miRNAs can affect the biological fate of endothelial cells. For example, Huang et al. confirmed that HepG2-derived vasorin (VASN, a type I transmembrane protein) can be effectively delivered into human umbilical vein endothelial cells (HUVECs) via receptor-dependent endocytosis of exosomes, which in turn induced migration of HUVECs [73]. In addition, the number of HepG2 exosomes was firmly associated with the degree of lumen formation [74]. Exosome-mediated miRNA transfer also modulates the biology of ECs. Lin et al. revealed that HCC cells secrete exosomes containing miR-210-3p (miR-210), which can promote the tube formation of ECs in vitro. Specifically, exosomal miR-210 can enhance angiogenesis by inhibiting the expression of SMAD4 and STAT6 in ECs [75]. 


\section{Promoting tumor angiogenesis}

In addition to EC tubulogenesis-related angiogenesis, exosomes are involved in other mechanisms promoting tumor angiogenesis. VEGF, as the most powerful proangiogenic cytokine, has the ability to transduce signals necessary for the formation of the three-dimensional vascular tube and in regulating vascular permeability. Conigliaro et al. found that CD90+-derived exosomes expressed high levels of lncRNA H19, which could substantially increase VEGF release and the production of VEGF-R1, hence stimulating angiogenesis. After lncRNA H19 silencing, the expression level of VEGF protein decreased significantly [76]. Therefore, exosomes could regulate the transcription of target cell-related genes and promote angiogenesis. In addition, exosomes are involved in the regulation of the HCC angiogenesis-related signaling pathway. The expression of miR-221 and miR-21 are elevated in HCC and HCC-derived exosomes [77]. miR221 can stimulate the activation of the SAND/NF-kB pathway and upregulate angiogenic factor CXCL16 expression, which plays a role in promoting angiogenesis [78]. miR-21 can activate the STAT3/VEGF signaling pathway and shape a vascular microenvironment for $\mathrm{HCC}$ [79]. As a response to this vascular microenvironment, HCC shows accelerated growth kinetics. These observations suggest the role of HCC-secreted exosomes in remodeling TME and promoting tumor angiogenesis.

\section{Exosome-mediated HCC invasion and metastasis: local} and distant microenvironments act as critical sanctuaries Metastasis is an important feature distinguishing tumors from non-neoplastic diseases. Before the occurrence of distant metastasis, tumor-secreted exosomes can activate invasive-related soluble factors to remold the local and distant TME for the implementation of tumor metastatic invasion.

\section{Exosomes and EMT}

Epithelial-mesenchymal transformation (EMT) is a reversible dedifferentiation process characterized by the loss of epithelial characteristics and the acquisition of typical mesenchymal properties, which is considered the central mechanism of HCC invasion and metastasis $[99,100]$. A recent study indicated that HCC cell-derived exosomes can increase HGF levels in the cell culture supernatant [80]. HGF has been reported to be the most potent growth factor for hepatocytes and its receptor. In the TME, HGF has been suggested to cause HCC phenotypical changes through EMT, migration, and invasion [101, 102]. Although not directly verified, there may be a correlation between HCC-derived exosomes and the induction of EMT. A recent study confirmed this correlation from the opposite perspective. The differential expression of exosome proteins from conditioned media of HCC cells was analyzed by Karaosmanoğlu and colleagues. These authors found that some specific proteins were highly enriched in exosomes secreted by slug-overexpressing HCC cells, which were involved in the induction of either partial or total EMT [81]. In addition, exosomes derived from CAFs could transfer miR-320a to HCC cells, which could suppress EMT and videlicet, while the loss of antitumor miR$320 \mathrm{a}$ in the exosomes of CAFs could induce EMT and promote tumor progression [69]. However, the role of exosomes in EMT initiation requires further elucidation.

\section{Exosome-mediated ECM remodeling promotes local tumor invasion}

Some major components of ECM include collagens, laminins, fibronectin, glycosaminoglycans, and proteoglycans, which play a very crucial role in changing the phenotypic and functional characteristics of HCC and stroma cells [103]. Exosomes can set up a mechanically stiff microenvironment by enhancing the reorganization of ECM, which ultimately promotes tumor metastasis. He et al. found that exosomes derived from highly metastatic HCC cell lines were packed with proteins such as met, caveolin, and S100. After ingestion by the normal liver cell line MIHC, these exosomes contribute to enhancing the migratory and invasive potential of MIHC. In addition, the expression of Akt, phosphorylated MEK1/2, and metalloproteinases (MMP-2 and MMP-9) was increased significantly [82]. Based on these data, HCC-derived exosomes trigger the production of MMPs and enable the degradation of ECM to energize local invasion and cell migration.

\section{Exosomes promote vascular leakage and benefit the engraftment of tumor cells}

Exosomes derived from tumors have attracted more attention for the conversion of vascular permeability, which ultimately leads to tumor metastatic dissemination. Cell-to-cell junctions between endothelial cells are dependent on VE-cadherin, catenin (p120-, p0071-, and $\beta$-catenin), ZO-1, and claudin-5 [104]. Recent evidence indicates that miR-103 derived from hepatoma cells could be transmitted to endothelial cells via exosomes, which then inhibit the expression of VE-cadherin, p120catenin (p120), and ZO-1 to attenuate the integrity of endothelial junctions, induce vascular leakiness, and consequently facilitate tumor metastasis [83]. Furthermore, clinical data show that high serum exosomal miR1247-3p levels are associated with lung metastasis in HCC patients. The specific mechanism by which exosomes facilitate tumor cell arrival to the lungs and achieve tumor engraftment is the conversion of fibroblasts. High-metastatic cells secrete exosomal miR-1247$3 p$ to convert fibroblasts to CAFs in the lung premetastatic niche by targeting B4GALT3 [50]. Taken together, 
these data suggest that the intrinsic interactions of tumor-derived exosomes with target cells in a tumor environment further illuminate new mechanisms of $\mathrm{HCC}$ expansion and metastasis.

\section{The therapeutic usage of exosomes in the HCC microenvironment}

As exosome-based communication in the HCC microenvironment can function as a determinant factor to promote the malignant behavior of cancer cells, exosomes in the HCC microenvironment may provide an opportunity for targeted therapeutic intervention to eventually prevent $\mathrm{HCC}$ and prolong the duration of survival for HCC patients. Because exosome-mediated immune regulation in TME plays an important role in the occurrence and development of $\mathrm{HCC}$, it is reasonable to expect that the exosome-based communication system is beneficial to stimulate the immune response and to mediate HCC immunotherapy. In addition, increasing interest is focused on the utility of exosomes as therapeutic tools for drug and biological molecule transfer.

\section{Exosome-based immunotherapy in the HCC microenvironment}

Enhancing immunity is the basic therapeutic approach to HCC therapy [105]. Exosomes have been shown to enhance tumor immunogenicity and antitumor immune responses. Rao et al. revealed that tumor cell-derived exosomes expressed an array of HCC antigens, which significantly activated the immune response mediated by dendritic cells (DCs) in a preclinical model, ultimately reshaping the HCC immune microenvironment and improving antitumor effects [59]. Moreover, DC-derived exosomes have been demonstrated to be useful as cancer vaccines for immunotherapy. A study using exosomes derived from $\alpha$-fetoprotein (AFP)-expressing DCs (DEXAFP) to explore the relationship between the tumor immune microenvironment and HCC suppression is ongoing. Benefitting from the enhanced CD8+ T lymphocyte response, the expression levels of IFN- $\gamma$ and IL-2 were improved, while CD25+Foxp3+ regulatory $\mathrm{T}$ (Treg) cells, IL-10, and TGF- $\beta$ levels were decreased in tumor sites. DC-derived exosomes can significantly impede HCC growth and prolong survival rates in mice [84]. In terms of exosome-mediated immunotherapy, another example showed that exosomes derived from HCC cells were enriched for stress-induced heat shock protein (HSP) to improve tumor immunogenicity and induce NK cell responses through the upregulation of inhibitory receptor CD94 and downregulation of activating receptors (CD69, NKG2D, and NKp44) [85]. Immune checkpoint protein inhibitors have revolutionized cancer treatment; however, anti-PD-L1/PD-1 therapy is only effective in $10-30 \%$ of patients [106]. A recent study showed that exosomes derived from tumors with PD-L1 could suppress T cell activation in the draining lymph node, suggesting that tumor growth could be suppressed by exosomal PD-L1 blockade. Inhibiting the release of PD-L1 exosomes might overcome resistance to current antibody approaches [86].

\section{Exosomes as therapeutic tools in the HCC microenvironment}

Accumulating evidence has shown that exosomes could present promising drugs and biological molecule delivery vehicles for cancer therapy due to biocompatibility and stability, endure modification, and cross the blood-brain barrier $[107,108]$. Mesenchymal stem cells (MSCs) produce massive exosomes. When adipose tissue-derived MSC (AMSC) exosomes were transfected with miR-122 expression plasmids, miR-122 can be effectively packaged into secreted exosomes and transferred to HCC cells, thereby rendering HCC cells sensitive to chemotherapy agents (5-FU and sorafenib) [71]. Another study provided a novel approach to package the contents into exosomes. Functional miR-26a was loaded via electroporation into the exosomes of engineered $293 \mathrm{~T}$ cells, and then, these engineered exosomes could bind selectively to HCC cells through the scavenger receptor to reduce the rates of cell migration and proliferation [87]. In an example of chemotherapeutic delivery, exosomes were used as targeted drug delivery vehicles for low immunogenicity and toxicity. Immature dendritic cells (imDCs) were engineered to express a fully characterized exosome membrane protein (Lamp2b) fused with $\mathrm{v}$ integrin-specific iRGD peptide for the targeted transportation of exosomes loaded with doxorubicin to tumor tissues [88]. Such targeted exosomes were injected intravenously, which specifically delivered doxorubicin to the tumor tissue, resulting in the inhibition of tumor growth without significant toxicity.

\section{The relationship between chemosensitivity and exosomes in the HCC microenvironment}

HCC has been indicated as resistant to traditional chemotherapeutic strategies. Although many unanswered questions remain, the association between exosomes and drug resistance has provided important insights into a promising new strategy for $\mathrm{HCC}$ chemoresistance. Studies have found that CD133+ T-IC [109] and the ATP-binding cassette family [110] are important factors leading to HCC drug resistance. Recent studies have shown that exosomes have been implicated in promoting resistance to chemotherapy by promoting the expression of CD133+ T-IC and ABCG2 in the TME. Takahashi and colleagues demonstrated that exosomal linc-ROR from HCC can be significantly upregulated after the incubation of tumor cells with diverse anticancer agents, thereby activating TGF- $\beta$ signaling and promoting colony formation of CD133+ T-IC, 
resulting in the reduction of cancer cells sensitive to chemotherapy [66]. In addition to the regulation of CD133+ T-IC by exosomal linc-ROR, another mechanism associated with $\mathrm{HCC}$ chemoresistance is the upregulation of ABCG2 by exosomal lincRNA-VLDLR. Based on these observations, RNAi-mediated knockdown of linc-VLDLR can reduce cell viability, arrest the cell cycle in the G1/S phase, and promote apoptosis [89]. Another in vitro study revealed that HGF/c-Met/Akt signaling pathways can be triggered by HCC cell-derived exosomes, which play an important role in restricting sorafenib-induced apoptosis and contribute to drug resistance of liver cancer [80]. These examples of exosome manipulation reveal complex mechanisms of chemoresistance in the TME and may offer potential therapeutic strategies for HCC.

\section{Conclusions}

TME is the cellular environment that malignant cells need for survival, growth, proliferation, and metastasis. Exosomes, as a novel intercellular communication mediator in the TME, have attracted attention for promoting tumor onset and progression. The present review summarized the role of exosomes in setting up and modifying the TME for the development and progression of HCC. Here, we revealed the specific mechanisms through which exosome-based communication within the HCC microenvironment promoted the malignant behavior of cancer cells. It is possible that exosome-mediated therapy in HCC patients could involve the multifaceted roles of exosomes in the TME. Future work is needed to illuminate the potential mechanisms by which exosomes have effector functions in the TME to provide an opportunity for targeted therapeutic intervention based on exosome communication systems.

\begin{abstract}
Abbreviations
CAFs: Cancer-associated fibroblasts; DCs: Dendritic cells; ECM: Extracellular matrix; ECs: Endothelial cells; EMT: Epithelial-mesenchymal transformation; EVs: Extracellular vesicles; FGF: Fibroblast growth factor; HCC: Hepatocellular carcinoma; HGF: Hepatocyte growth factor; HIF: Hypoxia-inducible factor; HSCs: Hepatic stellate cells; HSP: Heat shock protein; HUVECs: Human umbilical vein endothelial cells; IGF: Insulin-like growth factor; ILVs: Intraluminal vesicles; MMPs: Matrix metalloproteinases; MSCs: Mesenchymal stem cells; MVBs: Multivesicular bodies; PDK1: Pyruvate dehydrogenase kinase isozyme 1; TAMs: Tumor-associated macrophages; TGF- $\beta$ : Transforming growth factor- $\beta$; TILs: Tumor-infiltrating T Iymphocytes; TME: Tumor microenvironment; VASN: Vasorin; VEGF: Vascular endothelial growth factor
\end{abstract}

\section{Acknowledgements}

We are grateful to Prof. Yu Hai-qing (Department of Internal Medicine, University of Missouri) for positive suggestions concerning the manuscript.

\section{Authors' contributions}

HT designed the review. QW drafted the manuscript and prepared the figures. $L Y Z, D D L$, and $X Z$ helped to modify the manuscript. All authors read and approved the final manuscript.

\section{Funding}

This work was supported by the National Natural Science Foundation of China (No. 81772193)

\section{Availability of data and materials}

The material supporting the conclusions of this review is included within the article.

Ethics approval and consent to participate

Not applicable.

\section{Consent for publication}

Not applicable.

\section{Competing interests}

The authors declare that they have no competing interests.

Received: 10 February 2019 Accepted: 19 May 2019

Published online: 29 May 2019

\section{References}

1. Quail DF, Joyce JA. Microenvironmental regulation of tumour progression and metastasis. Nat Med. 2013;19:1423-37.

2. Junttila MR, Sauvage FJD. Influence of tumour micro-environment heterogeneity on therapeutic response. Nature. 2013;501:346-54.

3. Farazi PA, Depinho RA. Hepatocellular carcinoma pathogenesis: from genes to environment. Nat Rev Cancer. 2006:6:674-87.

4. Bissell MJ, Hines WC. Why don't we get more cancer? A proposed role of the microenvironment in restraining cancer progression. Nat Med. 2011;17: $320-9$.

5. Becker A, Thakur BK, Weiss JM, Kim HS, Peinado H, Lyden D. Extracellular vesicles in cancer: cell-to-cell mediators of metastasis. Cancer Cell. 2016;30: 836-48.

6. Kalluri R. The biology and function of exosomes in cancer. J Clin Invest. 2016;126:1208.

7. Pan BT, Johnstone RM. Fate of the transferrin receptor during maturation of sheep reticulocytes in vitro: selective externalization of the receptor. Cell. 1983;33:967-78

8. Cocucci E, Meldolesi J. Ectosomes and exosomes: shedding the confusion between extracellular vesicles. Trends Cell Biol. 2015:25:364-72.

9. Pfeffer SR. Unsolved mysteries in membrane traffic. Annu Rev of Biochem. 2007;76:629

10. Kosaka N. Decoding the secret of cancer by means of extracellular vesicles. I Clin Med. 2016:5:22

11. Record M, Carayon K, Fau - Poirot M, Poirot M, Fau - Silvente-Poirot S, Silvente-Poirot S. Exosomes as new vesicular lipid transporters involved in cell-cell communication and various pathophysiologies. Biochim Biophys Acta. 2014:108-20.

12. Takayuki K, Wen-Lang L, Yan IK, Chiara B, Tushar P. Intercellular nanovesiclemediated microRNA transfer: a mechanism of environmental modulation of hepatocellular cancer cell growth. Hepatology. 2011;54:1237-48.

13. Oosthuyzen W, Sime NEL, IVy JR, Turtle EJ, Street JM, Pound J, et al. Quantification of human urinary exosomes by nanoparticle tracking analysis. J Phys. 2014;229:S247-S47

14. Street JM, Barran PE, Mackay CL, Weidt S, Balmforth C, Walsh TS, et al. Identification and proteomic profiling of exosomes in human cerebrospinal fluid. Journal of Translational Medicine,10,1(2012-01-05). 2012:10:5.

15. Lässer C, Alikhani VS, Ekström K, Eldh M, Paredes PT, Bossios A, et al. Human saliva, plasma and breast milk exosomes contain RNA: uptake by macrophages. J Transl Med. 2011;9:9.

16. Bang C, Thum T. Exosomes: new players in cell-cell communication. Int J Biochem Cell Biol. 2012:44:2060-4.

17. Sun D, Fau ZX. Zhang S, Zhang S Fau - Deng Z-B, Deng Zb Fau - Grizzle W, Grizzle W Fau - Miller D, Miller D Fau - Zhang H-G, et al. Exosomes are endogenous nanoparticles that can deliver biological information between cells. Adv Drug Deliv Rev. 2013:342-7.

18. Vlassov AV, Magdaleno S, Setterquist R, Conrad R. Exosomes: current knowledge of their composition, biological functions, and diagnostic and therapeutic potentials. Biochim Biophys Acta. 2012;1820:940-8. 
19. Azmi AS, Bao B, Sarkar FH. Exosomes in cancer development, metastasis, and drug resistance: a comprehensive review. Cancer Metastasis Rev. 2013; 32:623-42.

20. Masyuk Al, Huang BQ, Ward CJ, Gradilone SA, Banales JM, Masyuk TV, et al. Biliary exosomes influence cholangiocyte regulatory mechanisms and proliferation through interaction with primary cilia. Am J Physiol Gastrointest Liver Physiol. 2010;299:990-9.

21. Zhong-Bin D, Xiaoying Z, Songwen J, Xiaoyu X, Jingyao M, Qilong W, et al. Intestinal mucus-derived nanoparticle-mediated activation of Wnt/ $\beta$-catenin signaling plays a role in induction of liver natural killer T cell anergy in mice. Hepatology. 2013;57:1250-61.

22. Fonsato V, Fau CF. Herrera MB, Herrera Mb Fau - Cavallari C, Cavallari C Fau Deregibus MC, Deregibus Mc Fau - Cisterna B, Cisterna B Fau - Bruno S, et al. Human liver stem cell-derived microvesicles inhibit hepatoma growth in SCID mice by delivering antitumour microRNAs. Stem Cells. 2012:1985-98.

23. Witek RP, Yang L, Liu R, Jung Y, Omenetti A, Syn WK, et al. Liver cell-derived microparticles activate hedgehog signaling and alter gene expression in hepatic endothelial cells. Gastroenterology. 2009;136:320-30 e2.

24. Wakim LM, Bevan MJ. Cross-dressed dendritic cells drive memory CD8+ Tcell activation after viral infection. Nature. 2011;471:629.

25. Greuter $\mathrm{T}$, Shah VH. Hepatic sinusoids in liver injury, inflammation, and fibrosis: new pathophysiological insights. J Gastroenterol. 2016;51:511-9.

26. Royo F, Schlangen K, Palomo L, Gonzalez E, Condevancells J, Berisa A, et al. Transcriptome of extracellular vesicles released by hepatocytes. Plos One. 2013;8:e68693

27. Hirsova P, Ibrahim SH, Krishnan A, Verma VK, Bronk SF, Werneburg NW, et al. Lipid-induced signaling causes release of inflammatory extracellular vesicles from hepatocytes. Gastroenterology. 2016;150:956-67.

28. Chahar HS, Bao X, Casola A. Exosomes and their role in the life cycle and pathogenesis of RNA viruses. Viruses. 2015;7:3204-25.

29. Kouwaki T, Okamoto M, Tsukamoto H, Fukushima Y, Oshiumi H. Extracellular vesicles deliver host and virus RNA and regulate innate immune response. Int J Mol Sci. 2017;18:666

30. Momen-Heravi F, Saha B, Kodys K, Catalano D, Satishchandran A, Szabo G. Increased number of circulating exosomes and their microRNA cargos are potential novel biomarkers in alcoholic hepatitis. J Transl Med. 2015;13:1-13.

31. Saha B, Momen-Heravi F, Kodys K, Szabo G. MicroRNA cargo of extracellular vesicles from alcohol-exposed monocytes signals naive monocytes to differentiate into M2 macrophages. J Biol Chem. 2016;291:149-59.

32. Li C, Alyssa C, Yu Z, Ruju C, Bo Y, Kitty A, et al. Epigenetic regulation of connective tissue growth factor by MicroRNA-214 delivery in exosomes from mouse or human hepatic stellate cells. Hepatology. 2014;59:1118-29.

33. El-Serag HB, Rudolph KL. Hepatocellular carcinoma: epidemiology and molecular carcinogenesis. Gastroenterology. 2007;132:2557-76.

34. Blum HE, Moradpour D. Hepatocellular carcinoma. N Engl J Med. 2011;365: $1118-27$.

35. Leonardi GC, Candido S, Cervello M, Nicolosi D, Raiti F, Travali S, et al. The tumour microenvironment in hepatocellular carcinoma (review). Int J Oncol. 2012;40:1733.

36. Feingold M, ., Hall BD, Lacassie Y, ., Martínez-Frías ML. Syndrome of microcephaly, facial and hand abnormalities, tracheoesophageal fistula, duodenal atresia, and developmental delay. Am J Med Genet A. 1997;69:571-582.

37. Hellerbrand C. Hepatic stellate cells - the pericytes in the liver. Pflügers Archiv:Eur J Physiol. 2013;465:775-8.

38. Amann T, Bataille F, Spruss T, Mühlbauer M, Gäbele E, Schölmerich J, et al. Activated hepatic stellate cells promote tumourigenicity of hepatocellular carcinoma. Cancer Sci. 2010;100:646-53.

39. Xiong S, Wang R, Chen Q, Luo J, Wang J, Zhao Z, et al. Cancer-associated fibroblasts promote stem cell-like properties of hepatocellular carcinoma cells through IL-6/STAT3/Notch signaling. Am J Cancer Res. 2018;8:302-16.

40. Park SY, Jeong KJ, Panupinthu N, Yu S, Lee J, Han JW, et al. Lysophosphatidic acid augments human hepatocellular carcinoma cell invasion through LPA1 receptor and MMP-9 expression. Oncogene. 2011;30:1351-9.

41. Gajewski TF, Schreiber H, Fau - Fu Y-X, Fu YX. Innate and adaptive immune cells in the tumour microenvironment. Nat Immunol. 2013:1014-22.

42. Zamarron BF, Chen W. Dual roles of immune cells and their factors in cancer development and progression. Int J Biol Sci. 2011;7:651-8.

43. Zhang S, Sun WY, Wu JJ, Wei W. TGF-beta signaling pathway as a pharmacological target in liver diseases. Pharmacol Res. 2014;85:15-22.

44. Postic C, Dentin R, Girard J. Role of the liver in the control of carbohydrate and lipid homeostasis. Diabetes Metab. 2004;30:398-408.
45. Lippolis C, Refolo MG, D'Alessandro R, Carella N, Messa C, Cavallini A, et al. Resistance to multikinase inhibitor actions mediated by insulin like growth factor-1. J Exp Clin Cancer Res. 2015;34:90.

46. Streuli $\mathrm{CH}$, Akhtar N. Signal co-operation between integrins and other receptor systems. Biochem J. 2009;418:491-506.

47. Hanahan D, Coussens L. Accessories to the crime: functions of cells recruited to the tumour microenvironment. Cancer Cell. 2012;21:309-22.

48. Pengfei L, Weaver VM, Zena $W$. The extracellular matrix: a dynamic niche in cancer progression. J Cell Biol. 2012;196:395.

49. Chen J, Wang Q, Xh HX, Chen X, Cao L, Chen L, et al. Involvement of PI3K/PTEN/AKT/mTOR pathway in invasion and metastasis in hepatocellular carcinoma: association with MMP-9. Hepatol Res. 2010;39: 177-86.

50. Fang T, Lv H, Lv G, Li T, Wang C, Han Q, et al. Tumour-derived exosomal miR-1247-3p induces cancer-associated fibroblast activation to foster lung metastasis of liver cancer. Nat Commun. 2018;9:191.

51. María M, Cristina GV, Carolina VB, Susana G, Fátima SC, Ángel GM, et al. Unidirectional transfer of microRNA-loaded exosomes from $T$ cells to antigen-presenting cells. Nat Commun. 2011;2:282

52. Moris D, Lu L, Qian S. Mechanisms of liver-induced tolerance. Curr Opin Organ Transplant. 2017;22:71

53. Yang N, Li S, Li G, Zhang S, Tang X, Ni S, et al. The role of extracellular vesicles in mediating progression, metastasis and potential treatment of hepatocellular carcinoma. Oncotarget. 2016;8:3683.

54. Pardee AD, Butterfield LH. Immunotherapy of hepatocellular carcinoma: unique challenges and clinical opportunities. Oncoimmunology. 2012;1:48-55.

55. Wang X, Shen H, Zhangyuan G, Huang R, Zhang W, He Q, et al. 14-3-3zeta delivered by hepatocellular carcinoma-derived exosomes impaired antitumour function of tumour-infiltrating T lymphocytes. Cell Death Dis. 2018:159.

56. Smyth MJ, Jeremy S, Erika C, Nadeen Z, Yokoyama WM, Yoshihiro H. NKG2D function protects the host from tumour initiation. J Exp Med. 2005:202:583-8.

57. Nadia G, Xim TY, Joncker NT, Augustine C, Fermin G, Na X, et al. NKG2Ddeficient mice are defective in tumour surveillance in models of spontaneous malignancy. Immunity. 2008;28:571-80.

58. Omodele A, Philippe B, Lola FM, Sonia AG, Skepper JN, Mar VG, et al. Natural killer cell cytotoxicity is suppressed by exposure to the human NKG2D ligand MICA*008 that is shed by tumour cells in exosomes. Cancer Res. 2010;70:481-9.

59. Rao Q, Zuo B, Lu Z, Gao X, You A, Wu C, et al. Tumour-derived exosomes elicit tumour suppression in murine hepatocellular carcinoma models and humans in vitro. Hepatology. 2016;64:456-72.

60. Chow A, Zhou W, Liu L, Fong MY, Champer J, Van Haute D, et al. Macrophage immunomodulation by breast cancer-derived exosomes requires toll-like receptor 2-mediated activation of NF-kappaB. Sci Rep. 2014;4:5750.

61. Costa-Silva B, Aiello NM, Ocean AJ, Singh S, Zhang H, Thakur BK, et al. Pancreatic cancer exosomes initiate pre-metastatic niche formation in the liver. Nat Cell Biol. 2015;17:816-26.

62. Yu L. Exosomes from melatonin treated hepatocellularcarcinoma cells alter the immunosupression status through STAT3 pathway in macrophages. Int J Biol Sci. 2017;13:723-34.

63. Xiong L, Zhen S, Yu Q, Gong Z. HCV-E2 inhibits hepatocellular carcinoma metastasis by stimulating mast cells to secrete exosomal shuttle microRNAs. Oncol Lett. 2017;14:2141-6.

64. Aucher A, Rudnicka D, Davis DM. MicroRNAs transfer from human macrophages to hepato-carcinoma cells and inhibit proliferation. J Immunol. 2013;191:6250-60

65. Kenji T, Yan IK, Hiroaki H, Tushar P. Modulation of hypoxia-signaling pathways by extracellular linc-RoR. J Cell Sci. 2014;127:1585-94

66. Takahashi K, Yan IK, Kogure T, Haga H, Patel T. Extracellular vesicle-mediated transfer of long non-coding RNA ROR modulates chemosensitivity in human hepatocellular cancer. FEBS Open Bio. 2014:458-67.

67. Chao C, Fei L, Liu X, Lu L, Hui X, Yang Q, et al. NF-kB-regulated exosomal miR-155 promotes the inflammation associated with arsenite carcinogenesis. Cancer Lett. 2017;388:21-33

68. Fu XT, Shi YH, Zhou J, Peng YF, Liu WR, Shi GM, et al. MicroRNA-30a suppresses autophagy-mediated anoikis resistance and metastasis in hepatocellular carcinoma. Cancer Lett. 2018;412:53763-79.

69. Zhang Z, Xiao L, Wei S, Yue S, Yang J, Li J, et al. Loss of exosomal miR-320a from cancer-associated fibroblasts contributes to HCC proliferation and metastasis. Cancer Lett. 2017;397:33-42. 
70. Sudarshana B, Bhattacharyya SN. Insulin-like growth factor-1 prevents miR-122 production in neighbouring cells to curtail its intercellular transfer to ensure proliferation of human hepatoma cells. Nucleic Acids Res. 2014;42:7170-85.

71. Lou G, Song X, Yang F, Wu S, Wang J, Chen Z, et al. Exosomes derived from miR-122-modified adipose tissue-derived MSCs increase chemosensitivity of hepatocellular carcinoma. J Hematol Oncol. 2015;8:122.

72. Tang J, Li Y, Liu K, Zhu Q, Yang WH, Xiong LK, et al. Exosomal miR-9-3p suppresses HBGF-5 expression and is a functional biomarker in hepatocellular carcinoma. Minerva Medica. 2018;109.

73. Aixue $H$, Jie D, Shaohua L, Chaonan W, Hongmei D, Hui L, et al. Exosomal transfer of vasorin expressed in hepatocellular carcinoma cells promotes migration of human umbilical vein endothelial cells. Int J Biol Sci. 2015;11:961-9.

74. Yukawa H, Suzuki K, Aoki K, Arimoto T, Yasui T, Kaji N, et al. Imaging of angiogenesis of human umbilical vein endothelial cells by uptake of exosomes secreted from hepatocellular carcinoma cells. Sci Rep. 2018;8.

75. Lin XJ, Fang JH, Yang XJ, Zhang C, Yuan Y, Zheng L, et al. Hepatocellular carcinoma cell-secreted exosomal microRNA-210 promotes angiogenesis in vitro and in vivo. Mol Ther Nucleic Acids. 2018:243-52.

76. Conigliaro A, Costa V, Dico AL, Saieva L, Buccheri S, Dieli F, et al. CD90+ liver cancer cells modulate endothelial cell phenotype through the release of exosomes containing H19 IncRNA. Molecular Cancer. 2015;14:155.

77. Wu Z, Zeng Q, Cao K, Sun Y. Exosomes: small vesicles with big roles in hepatocellular carcinoma. Oncotarget. 2016;7:60687-97.

78. Prasanna Kumar S, Das SK, Rachel G, Dong C, Jyoti S, Chadia R, et al. Multifunction protein staphylococcal nuclease domain containing 1 (SND1) promotes tumour angiogenesis in human hepatocellular carcinoma through novel pathway that involves nuclear factor $\mathrm{KB}$ and miR-221. J Biol Chem. 2012:287:13952

79. Ning Z, Wei-Dong D, Jian-Jun L, Liang Z, Xing W, Yin-Zhe X, et al. STAT3 regulates the migration and invasion of a stem-like subpopulation through microRNA-21 and multiple targets in hepatocellular carcinoma. Oncol Reports. 2015;33:1493-8.

80. Zhen $\mathrm{Q}, \mathrm{Wu}$ J, Wu J, Luo D, Jiang C, Ding Y. Exosomes derived from HCC cells induce sorafenib resistance in hepatocellular carcinoma both in vivo and in vitro. J Exp Clin Cancer Res. 2016;35:159.

81. Karaosmanoğlu $\mathrm{O}$, Banerjee $\mathrm{S}$, Sivas H. Identification of biomarkers associated with partial epithelial to mesenchymal transition in the secretome of slug over-expressing hepatocellular carcinoma cells. Cell Oncol. 2018:1-15.

82. Mian H, Hao Q, Poon TCW, Siu-Ching S, Xiaofan D, Ngai Na C, et al. Hepatocellular carcinoma-derived exosomes promote motility of immortalized hepatocyte through transfer of oncogenic proteins and RNAs. Carcinogenesis. 2015;36:1008-18.

83. Fang JH, Zhang ZJ, Shang LR, Luo YW, Lin Y, Yuan Y, et al. Hepatoma cellsecreted exosomal microRNA-103 increases vascular permeability and promotes metastasis by targeting junction proteins. Hepatology. 2018

84. Lu Z, Zuo B, Jing R, Gao X, Rao Q, Liu Z, et al. Dendritic cell-derived exosomes elicit tumour regression in autochthonous hepatocellular carcinoma mouse models. J Hepatol. 2017;67:739-48.

85. Lv LH, Wan YL, Lin Y, Zhang W, Yang M, Li GL, et al. Anticancer drugs cause release of exosomes with heat shock proteins from human hepatocellular carcinoma cells that elicit effective natural killer cell antitumour responses in vitro. J Biol Chem. 2012;287:15874-85

86. Poggio M, Hu T, Pai CC, Chu B, Belair CD, Chang A, et al. Suppression of exosomal PD-L1 induces systemic anti-tumour immunity and memory. Cell. 2019;177:414-27 e13.

87. Liang G, Kan S, Zhu Y, Feng S, Feng W, Gao S. Engineered exosomemediated delivery of functionally active miR-26a and its enhanced suppression effect in HepG2 cells. Int J Nanomedicine. 2018;13:585-99.

88. Tian Y, Li S, Song J, Ji T, Zhu M, Anderson GJ, et al. A doxorubicin delivery platform using engineered natural membrane vesicle exosomes for targeted tumour therapy. Biomaterials. 2014;35:2383-90.

89. Takahashi K, Yan IK, Wood J, Haga H, Patel T. Involvement of extracellular vesicle long noncoding RNA (linc-VLDLR) in tumour cell responses to chemotherapy. Mol Cancer Res. 2014;12:1377-87.

90. Balamurugan K. HIF-1 at the crossroads of hypoxia, inflammation, and cancer. Int J Cancer J Int Du Cancer. 2016;138:1058-66.

91. Bissell DM, Roulot D, George J. Transforming growth factor beta and the liver. Hepatology. 2001;34:859-67.
92. Yoshida K, Matsuzaki K, Murata M, Yamaguchi T, Suwa K, Okazaki K. Clinicopathological importance of TGF- $\beta /$ phospho-smad signaling during human hepatic fibrocarcinogenesis. Cancers. 2018;10:183.

93. Seo W, Eun HS, Kim SY, Yi HS, Lee YS, Park SH, et al. Exosome-mediated activation of toll-like receptor 3 in stellate cells stimulates interleukin-17 production by $\gamma \delta$ T cells in liver fibrosis. Hepatology. 2016;64:616.

94. Xu QG, Yu J, Guo XG, Hou GJ, Yuan SX, Yang Y, et al. IL-17A promotes the invasion-metastasis cascade via theAKTpathway in hepatocellular carcinoma. Mol Oncol. 2018;12:936-52.

95. Liu Z, Tu K, Liu Q. Effects of microRNA-30a on migration, invasion and prognosis of hepatocellular carcinoma. Febs Lett. 2016:588:3089-97.

96. Chen J, Yue Y, Shu L, Liu Y, Shu Z, Cao S, et al. MicroRNA-30a ameliorates hepatic fibrosis by inhibiting Beclin1-mediated autophagy. J Cell Mol Med. 2017;21.

97. Wei-Chih T, Paul Wei-Che H, Tsung-Ching L, Gar-Yang C, Ching-Wen L, Chun-Ming C, et al. MicroRNA-122, a tumour suppressor microRNA that regulates intrahepatic metastasis of hepatocellular carcinoma. Hepatology. 2010;49:1571-82.

98. Potente M, Gerhardt H, Carmeliet P. Basic and therapeutic aspects of angiogenesis. Cell. 2011;146:873-87.

99. Samy L, Jian X, Rik D. Molecular mechanisms of epithelial-mesenchymal transition. Nat Rev Mol Cell Biol. 2014;15:178-96.

100. Muh-Hwa Y, Chih-Li C, Gar-Yang C, Shih-Hwa C, Chien-Wei S, Teh-Ying $\mathrm{C}$, et al. Comprehensive analysis of the independent effect of twist and snail in promoting metastasis of hepatocellular carcinoma. Hepatology. 2010;50:1464-74.

101. Roussos ET, Condeelis JS, Patsialou A. Chemotaxis in cancer. Nat Rev Cancer. 2011;11:573-87

102. Whittaker S, Marais R Fau - Zhu AX, Zhu AX. The role of signaling pathways in the development and treatment of hepatocellular carcinoma. Oncogene. 2010:4989-5005.

103. Goessler UR, Hörmann K, Riedel F. Tissue engineering with chondrocytes and function of the extracellular matrix (review). Int J Mol Med. 2004;13:505.

104. Bazzoni G, Dejana E. Endothelial cell-to-cell junctions: molecular organization and role in vascular homeostasis. Physiol Rev. 2004;84:869-901.

105. Robbins PD, Morelli AE. Regulation of immune responses by extracellular vesicles. Nat Rev Immunol. 2014;14:195-208.

106. Page DB, Postow MA, Callahan MK, Allison JP, Wolchok JD. Immune modulation in cancer with antibodies. Annu Rev Med. 2014;65:185-202.

107. Yang T, Martin P, Fogarty B, Brown A, Schurman K, Phipps R, et al. Exosome delivered anticancer drugs across the blood-brain barrier for brain cancer therapy in Danio rerio. Pharm Res. 2015;32:2003-14.

108. Ha D, Yang N, Nadithe V. Exosomes as therapeutic drug carriers and delivery vehicles across biological membranes: current perspectives and future challenges. Acta Pharm Sin B. 2016;6:287-96.

109. Vlashi E, Pajonk F. Cancer stem cells, cancer cell plasticity and radiation therapy. Semin Cancer Biol. 2015;31:28-35.

110. Zhaolin C, Tianlu S, Lei Z, Pengli Z, Mingying D, Cheng $H$, et al. Mammalian drug efflux transporters of the ATP binding cassette (ABC) family in multidrug resistance: a review of the past decade. Cancer Lett. 2016:370:153-64

\section{Publisher's Note}

Springer Nature remains neutral with regard to jurisdictional claims in published maps and institutional affiliations.

Ready to submit your research? Choose BMC and benefit from:

- fast, convenient online submission

- thorough peer review by experienced researchers in your field

- rapid publication on acceptance

- support for research data, including large and complex data types

- gold Open Access which fosters wider collaboration and increased citations

- maximum visibility for your research: over $100 \mathrm{M}$ website views per year

At $\mathrm{BMC}$, research is always in progress.

Learn more biomedcentral.com/submission 